\title{
A Cardiac Cause of Exertional Dizziness
}

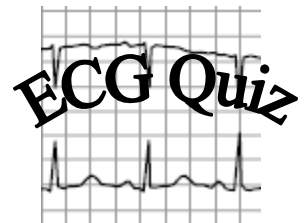
Bassem Chehab, M.D.

Peter Tadros, M.D.

University of Kansas Medical Center

Cardiovascular Research Institute

Cardiovascular Division

Kansas City, KS

A 22-year-old male without obvious previous medical history presented for recurrent episodes of dizziness during the last year. He noted that his dizziness worsened with exertion, mainly when he was running or working in his garage. His dizziness also worsened during the summer time, especially when he was in the sun. The initial electrocardiogram is below.

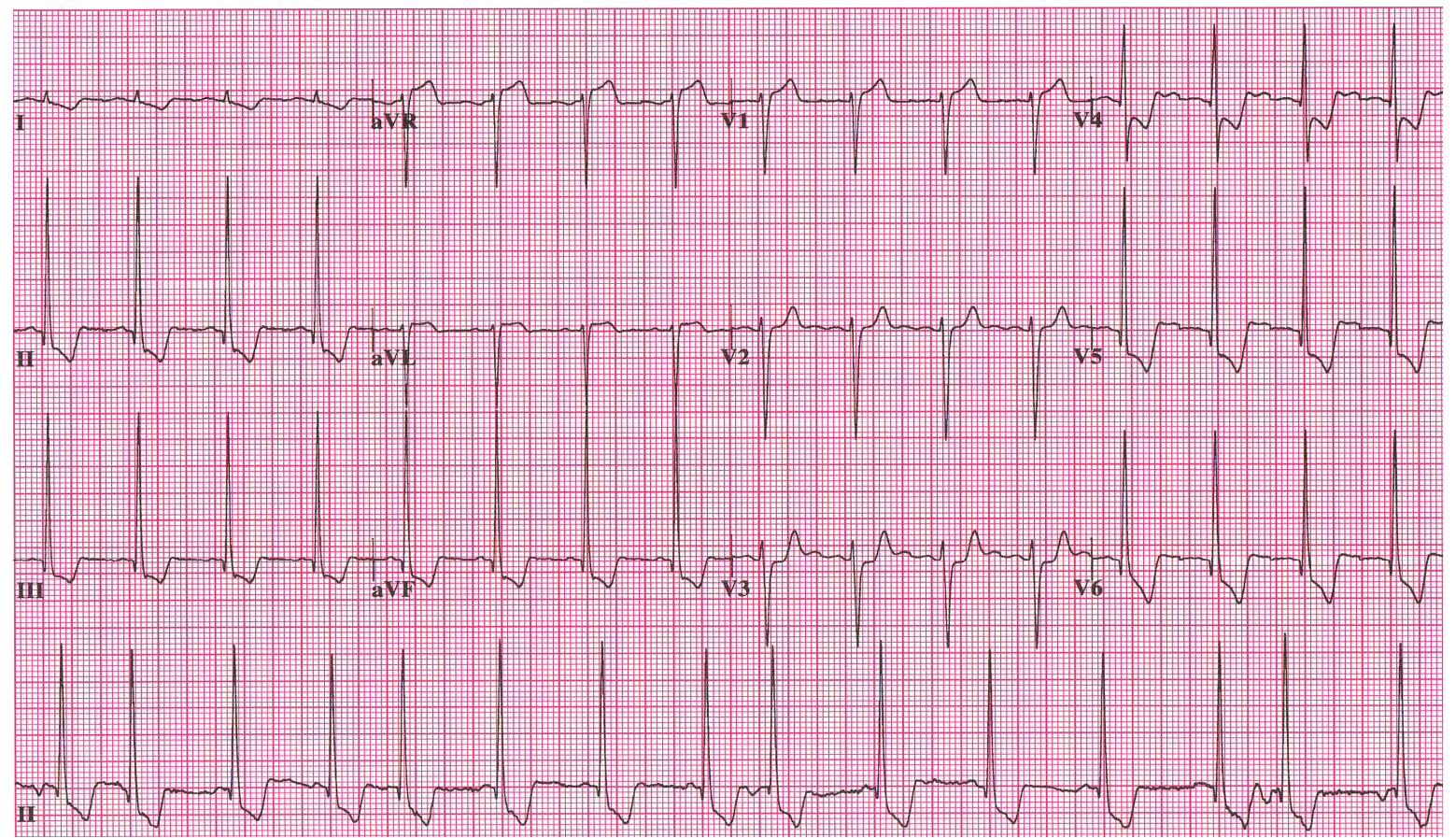

\section{What is the diagnosis?}

A) Severe aortic valve stenosis

B) Inferolateral wall infarction

C) Hypertrophic cardiomyopathy

D) Atrial fibrillation with atrioventricular block 


\section{CORRECT ANSWER: C}

The ECG reveals normal sinus rhythm with normally conducted atrial premature beats (beats $2,5,9$, and 14). Left ventricular hypertrophy is seen ( $\mathrm{S}$ wave in $\mathrm{aVR} \geq 15 \mathrm{~mm}$; $\mathrm{R}$ wave in aVF $\geq$ $21 \mathrm{~mm}$; $\mathrm{R}$ wave in V5 $+\mathrm{S}$ wave in V6 $\geq 20 \mathrm{~mm}$ ) with associated ST-T abnormalities are evident (marked $\mathrm{T}$ wave inversion are noted thought the leads). A marked increase in QRS voltage and ST-T abnormalities with severe $\mathrm{T}$ wave inversion in a young patient with dizziness is highly suggestive of hypertrophic cardiomyopathy (the anterior and anterolateral Q waves are secondary to hypertrophic cardiomyopathy, not previous infarction). This patient underwent an echocardiogram revealing asymmetric septal hypertrophy with dynamic left ventricular outflow tract gradient (resting gradient $40 \mathrm{mmHg}$, post Valsalva gradient of $112 \mathrm{mmHg}$ ) consistent with the diagnosis of hypertrophic cardiomyopathy.

\section{Discussion}

Hypertrophic cardiomyopathy $(\mathrm{HCM})$ is an uncommon genetic disorder that typically is inherited in an autosomal dominant fashion with variable penetrance and expressivity. ${ }^{1}$ It is characterized by altered myocyte shape, size, and alignment, which along with increased myocardial fibrosis, results in marked ventricular hypertrophy, stiffness, and diastolic dysfunction. The vast majority of patients have abnormal ECGs with LVH in 50-65\%, left atrial abnormalities in 20-40\%, and pathological Q wave (especially leads I, aVL, V4-V5) in 20-30\%. ST and T wave changes (repolarization changes secondary to left ventricular hypertrophy) are the most common ECG findings, while right axis deviation is rare. ${ }^{2}$ The most frequent cause of mortality is sudden cardiac death, with risk factors including young age and a history of syncope and/or asymptomatic ventricular tachycardia on ambulatory monitoring. ${ }^{1,2}$ Dizziness is common in patients with HCM with elevated pressure gradients across the left ventricular outflow tract. It is worsened by exertion and may be exacerbated by hypovolemia following high levels of exertion or increased insensible fluid loss (e.g., during extreme heat). ${ }^{2}$

\section{References}

${ }^{1}$ Wynne J, Braunwald E. The Cardiomyopathies. In: Zipes DP, Libby P, Bonow RO, Braunwald E (Eds.), Braunwald's Heart Disease. $7^{\text {th }}$ Edition. Philadelphia, PA: Elsevier Saunders, 2005:1659. ISBN 0-7216-0509-2.

2 Surawicz B, Knilans T. Chou's Electrocardiography in Clinical Practice: Adult and Pediatric. $6^{\text {th }}$ ed. Philadelphia, PA: WB Saunders, 2008. ISBN 978-1-4160-3774-3.

Keywords: hypertrophic cardiomyopathy, electrocardiography, dizziness 